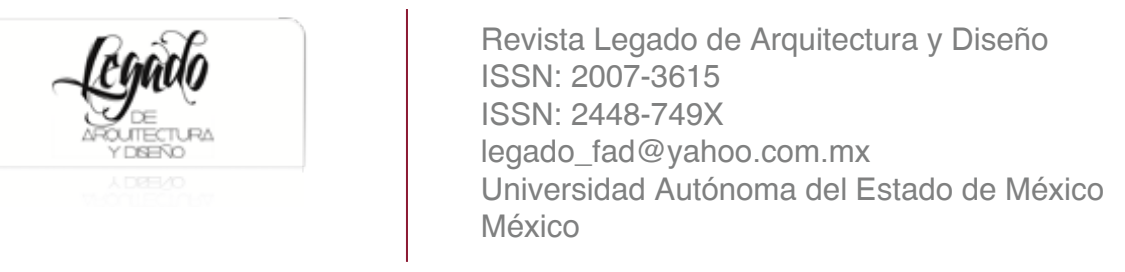

\title{
EL ESPACIO ARQUITECTÓNICO Y LA OPRESIÓN DE LA MUJER EN LA VIVIENDA MEXICANA
}

\author{
Villarreal-Pimienta, Laura Astrid; Ríos-Llamas, Carlos \\ EL ESPACIO ARQUITECTÓNICO Y LA OPRESIÓN DE LA MUJER EN LA VIVIENDA MEXICANA \\ Revista Legado de Arquitectura y Diseño, vol. 15, núm. 27, 2020 \\ Universidad Autónoma del Estado de México, México \\ Disponible en: http://www.redalyc.org/articulo.oa?id=477963263006
}

Esta obra está bajo una Licencia Creative Commons Atribución-NoComercial-SinDerivar 4.0 Internacional. 


\title{
EL ESPACIO ARQUITECTÓNICO Y LA OPRESIÓN DE LA MUJER EN LA VIVIENDA MEXICANA
}

\author{
ARCHITECTURAL SPACE AND THE OPPRESSION \\ OF WOMEN IN MEXICAN HOUSES
}

\author{
Laura Astrid Villarreal-Pimienta pimientastrid@gmail.com \\ Universidad De La Salle Bajío, México \\ Carlos Ríos-Llamas carlosrios@delasalle.edu.mx \\ Universidad De La Salle Bajío, México
} Revista Legado de Arquitectura y Diseño,
vol. 15, núm. 27, 2020

Universidad Autónoma del Estado de México, México

Recepción: 12 Agosto 2019 Aprobación: 19 Noviembre 2020

Redalyc: http://www.redalyc.org/ articulo.oa?id $=477963263006$
Resumen: Entre las fronteras que separan a los hombres y a las mujeres, la arquitectura ocupa un papel primordial por la reproducción de las desigualdades y tensiones a partir de la asignación de roles y de usuarios a los diferentes espacios diseñados. Más allá de los estudios sobre la disparidad en el número de profesionistas de la arquitectura hombres y mujeres o el análisis del papel de las mujeres en la historia del arte y la arquitectura, conviene adentrarse en las interconexiones que atraviesan los espacios habitados y las problemáticas de género, es decir, evidenciar que la arquitectura reproduce y reconfigura subjetividades a través de las disimetrías en el espacio simbólico. Este documento introduce de manera teórica la reflexión sobre la mujer y las estructuras de dominación que se materializan en la obra arquitectónica contemporánea. Se revisan dos ejemplos de diseño de vivienda en México y se hace una reflexión crítica sobre cómo los hombres y las mujeres piensan, utilizan y representan sus espacios cotidianos. Con el análisis, un doble componente, el espacio vivido y la tipología de la vivienda, se establecen una serie de cuestionamientos sobre las relaciones entre la vivienda, la manera de pensar y de vivir de las mujeres, así como el horizonte jerárquico y patriarcal con el que se diseñan y construyen los espacios domésticos del México contemporáneo.

Palabras clave: arquitectura, desigualdades, feminismo, vivienda.

Abstract: Among the borders separating men and women, architecture occupies a primary role on the reproduction of inequalities and tensions, by assigning roles and users to different spaces. Often, the studies focus on inequalities between men and women in the field of architecture; others have chosen an historical approach to reveal the participation of women in the history of art and architecture. However, it is also important to explore the gender differences inhabiting architectural spaces. Architecture reproduces and reconfigures subjectivities through asymmetries in symbolic space. This paper introduces the discussion on women and the structures of domination expressed in contemporary architectural work and its material realm. Two examples of housing design in Mexico are reviewed and analyzed, pointing at the different ways in which men and women think, use and represent their daily spaces. The analysis is structured on a double component: the lived space and the typology of the dwelling. Then, a series of questions are established about the relationships between dwelling, the way of thinking and living of women, as well as the hierarchical and patriarchal horizon with the one that designs and builds the domestic spaces of contemporary Mexico.

Keywords: architecture, inequalities, feminism, housing.

\section{INTRODUCCIÓN}

Aunque es posible identificar con claridad las consecuencias de la diferenciación de género en el conjunto de la historia del arte y en la 
filosofía, los estudios que se refieren de manera particular a la arquitectura como promotora y reflejo de las construcciones sociales que manifiestan dichas diferencias siempre han aparecido como algo irrelevante (Hills, 2003: 4). Mientras que, en la pintura, por ejemplo, la construcción de una corporalidad femenina y de una serie de valores estéticos y culturales asociados es objeto de múltiples estudios, ${ }^{[1]}$ no existe por otra parte una plataforma teórica que indague la complejidad de la corporalidad y el espacio arquitectónico con la misma profundidad, lo que deriva en el cuestionamiento por la hegemonía masculina no solamente en lo que se refiere al diseño de los espacios sino a la construcción de los discursos.

La investigación entre la arquitectura de las ciudades y la perspectiva de género es una línea de investigación novedosa y con importante producción de reflexiones en distintos campos como historia, arqueología, arte, antropología y sociología (Martínez-López, 2016: 11). A la pregunta sobre cuáles serían las implicaciones de la arquitectura en términos de género, habría que responder afirmando que "la arquitectura siempre ha sido utilizada para acentuar las diferencias sociales y establecer identidades" (Kuhlmann, 2013: 202). Judith Butler aborda la manera en que las suposiciones acerca del género normativo restringen no sólo la descripción que tenemos de lo humano, sino también de lo vivible (Butler, 1990: 26), en este caso, de la habitabilidad. En este sentido, las relaciones que se establecen entre la arquitectura y las perspectivas teóricometodológicas del diseño arquitectónico impactan necesariamente sobre cómo se construyen las ideas y las relaciones entre la propia construcción del usuario y el espacio que habita.

Es importante mencionar que el grueso de las reflexiones del feminismo sobre la relación de género y arquitectura corresponde principalmente a las últimas décadas del siglo XX. No obstante, varios autores han puesto en tensión esta grave carencia: comenzando primero por la crítica de las feministas materialistas en la revolución industrial, pasando luego por las pioneras del movimiento moderno, para llegar hasta las teóricas del urbanismo de los años 70 como Jane Jacobs o la excepcional Denise Scott Brown. De manera más reciente existen muchas iniciativas que reflexionan y hacen propuestas sobre la arquitectura de género como un área específica de las teorías del diseño y la edificación, aunque todavía no se puede afirmar que sus discursos se hayan incorporado transversalmente en el del establishment oficial de la disciplina (Novas, 2014: 12). Desde los primeros años de 1990 surgieron algunos de los posicionamientos más radicales del feminismo haciendo un llamado a revertir el modelo patriarcal de la profesión arquitectónica para introducir una nueva práctica más femenina que se materializaría en los procesos de diseño (Rendell, 2011:18). No obstante, como indica Novas recuperando la voz de Hernández, "ni siquiera el rencor postmoderno hacia los postulados más discutidos de [...] grandes arquitectos, habría permitido consentir, en las puertas del siglo XXI, la hipótesis temeraria de admitir entre los arquitectos y urbanistas que la ausencia de la lectura de género es una de las mayores faltas en la teoría del conocimiento arquitectónico de este siglo (2014: 11). 
Los debates del feminismo sobre la arquitectura se fueron multiplicando hasta principios del siglo XXI, pero hasta la fecha apenas se perfila una teoría de la arquitectura que integre los valores del feminismo más allá del discurso sobre el papel de la mujer en el conjunto de la historia del arte y en los bajos índices de participación femenina en el entorno de los profesionistas y famosos arquitectos contemporáneos. Se habla de reparar el papel que juega el colectivo femenino, grupo que representa la mitad de la población mundial y que durante siglos ha estado al margen de la práctica y teoría arquitectónica, como un requisito básico para una sociedad más justa (Novas, 2014: 11), pero a veces la arquitectura de género se aborda solamente como una reformulación de los análisis sociales, culturales y espaciales desde la mirada femenina.

Esto obedece, de acuerdo con Kulhmann, a que la teoría de la arquitectura se sustenta en un conjunto de valores y estándares que, si bien se han estado modificando a través de las diferentes épocas y estilos, mantienen una tradición masculina que es prácticamente invariable en lo que se refiere a la configuración de conceptos; esto se evidencia de manera más explícita en que "las teorías de las proporciones y las convenciones estéticas [hasta la modernidad] han reflejado casi exclusivamente valores masculinos" (2013: 202).

Si uno de los grandes méritos del feminismo ha sido el rescate de los múltiples escenarios sociales y políticos desde la condición particular de las mujeres, no es raro que las perspectivas marxistas sobre los sistemas de dominación y la disimetría de las relaciones entre hombres y mujeres hayan cobrado importancia como marco explicativo y productor de nuevas perspectivas. Por otro lado, "la influencia de las metodologías marxistas en la historia de la arquitectura ha jugado un rol fundamental para criticar el rol que la historia del arte le ha dado al diseñador poniéndolo en el centro de la disciplina" (Rendell, 2011:38).

Fiel a la metodología del materialismo histórico, que permite la reflexión sobre la arquitectura y el género desde la producción social del espacio, este análisis se concentra por un lado en los roles de género que se manifiestan en el contexto cultural de la construcción, y por el otro, en las representaciones y las maneras de habitar en los espacios domésticos. Se considera, como punto de partida, que el arquitecto es un agente fundamental en la producción sociocultural del espacio, y que lejos de adherirse a las tradiciones hegemónicas y sus dicotomías sexuadas de la vivienda, puede convertirse en un productor de nuevos tejidos sociales y espaciales que articulen hombres y mujeres desde la equidad frente a la cultura, los recursos materiales y la gestión del poder.

Ya desde 1990, Butler insistió en el género como una construcción social, y planteaba la pregunta sobre "si el género es una construcción, ¿podría construirse de distinta manera, o acaso su construcción conlleva alguna forma de determinismo social que niega la posibilidad de que el agente actúe y cambie?" (1990: 56). 


\section{METODOLOGÍA}

Diversas metodologías han propuesto un abordaje de las desigualdades entre hombres y mujeres con respecto al espacio edificado. En este caso particular, el enfoque metodológico se compone de dos dimensiones principales: el espacio vivido y las tipologías de vivienda (Lawrence, 1982). De esta manera, se ponen en discusión, tanto el espacio simbólico como las intervenciones arquitectónicas a partir de la configuración de las casas y modulación de lo doméstico. Desde esta perspectiva teórico-reflexiva, se puede plantear un estudio más concreto de la vivienda mexicana a partir de un análisis comparativo de la configuración del espacio doméstico contemporáneo desde la perspectiva del rol de la mujer, que parte del análisis de las distribuciones espaciales en varias tipologías de vivienda.

El objeto principal de estudio en este artículo es el diseño arquitectónico de la vivienda mexicana como factor de desigualdad de género. En el entendido de que "todos los espacios son potencial para la discriminación de género porque pueden ser mecanismos de exclusión, [... y que] inclusive un departamento o una casa unifamiliar constituye un espacio que está caracterizado por las diferencias de género que controlan más o menos los comportamientos de los ocupantes" (Kuhlmann, 2013: 173), se trata de realizar un análisis que ponga en evidencia los desequilibrios en la articulación del poder político y simbólico desplegadas en el diseño, la apropiación y uso de los distintos espacios arquitectónicos dispuestos en la vivienda mexicana en algunos ejemplos específicos, y desde un conjunto de voces más o menos representativas.

De esta manera, se hace un doble ejercicio para el análisis: en una primera parte se revisan y discuten los postulados sobre los que se construyen las maneras de habitar la vivienda mexicana, tomando como referencia algunas obras literarias que ejemplifican la representación de la feminidad y asignación de roles al espacio doméstico; la segunda parte propone un análisis de tipologías de la vivienda a partir de un par de casos de producción de vivienda social en serie: el Conjunto Residencial Cañada del Real, en la ciudad de León y el Conjunto Habitacional Las Anacuas en Monterrey, ambos en México. Estos proyectos de multifamiliares, con menos de 10 años de haberse concluido, se proponen como casos emblemáticos del espacio doméstico y la configuración de las viviendas contemporáneas del país.

A partir del análisis del espacio vivido y la tipología de la vivienda, este artículo propone un primer acercamiento al género como sistema de análisis arquitectónico de las dicotomías entre hombre/mujer y espacio exterior/interior; seguido de una revisión genealógica del complejo arquitectura y desigualdad de género para, finalmente, interpretar la adecuación de mobiliario y la asignación de roles e identidades a los espacios arquitectónicos del ambiente doméstico. 


\section{DISCUSIÓN}

\section{La dicotomia de sexos y de espacios: exterior/interior, hombre/mujer}

En primer lugar, es importante analizar la construcción de diferencias entre hombres y mujeres desde las relaciones que se asignan a los cuerpos con el espacio interior y exterior. Por lo que, se debe poner atención en la representación de los sujetos y de los cuerpos en arquitectura, para abordar el quehacer del diseño desde una perspectiva socioespacial que considera la representación simbólica con que se identifica los diferentes elementos geométricos y ambientales de los espacios proyectados y construidos.

La construcción teórico política que vinculaba las mujeres a lo doméstico y a los hombres a lo cívico tiene una larga historia en la teoría de la arquitectura y el urbanismo (Hill, 2003: 7). El feminismo hizo hincapié desde las últimas décadas del siglo XX en la asignación de la figura femenina a los espacios del "adentro" y al varón como el habitante por antonomasia del "afuera” (Ríos-Llamas, 2018b). Esta manera de identificar el espacio doméstico con la mujer es, por un lado, una consecuencia de la dominación masculina y las imposiciones canónicas de las estructuras familiares, y al mismo tiempo una proyección del espacio doméstico proyectado y materializado a partir de elementos determinantes de la actividad y permanencia de las mujeres en el hogar, que van desde la distribución espacial hasta la disposición de muebles y la asignación de tareas. Conviene, en este sentido, desdibujar el espacio público y exterior que excluye a las mujeres, y hacer un ejercicio crítico del espacio íntimo en sus alcances para la emancipación femenina.

En un ejercicio reflexivo sobre el complejo fear \& space en el que se suelen abordar los estudios de violencia de género y exclusión de la figura femenina en el espacio urbano, conviene pensar en aquellos lugares a los que las mujeres piensan que no deben estar o transitar solas, como los espacios deshabitados, los espacios oscuros, o los espacios directamente asociados a las actividades masculinas como algunos bares y terrazas, billares, o jardines y plazas públicas. En este sentido, se tiende a justificar la intimidad más como un elemento femenino de la vivienda desde el enfoque de lo cerrado por su antítesis con lo abierto entendido como un escenario donde se acentúan el miedo y la violencia. El miedo (a lo cerrado y a lo abierto) se experimenta con mayor intensidad en las calles porque las subjetividades se amplían y se diversifican (Ríos-Llamas, 2018a). En cuanto a las relaciones dentro/fuera que se transfieren al dualismo mujer/ varón, es el sujeto masculino el que representa la amenaza hacia el sujeto femenino ante las agresiones sistemáticas perpetradas por hombres en el uso de las calles. Las amenazas hacia las mujeres van desde un silbido, un piropo, un acercamiento sexual, miradas hostiles, comportamientos corporales invasivos. La consecuencia de esta serie de comportamientos deriva en la exclusión de la mujer del espacio urbano y en el refuerzo de las representaciones del espacio doméstico como un cobijo a la violencia materializada en el afuera de las ciudades. 
Además, lo privado como sistema de representación social, se materializa en las formas arquitectónicas convertidas en un instrumento de opresión de las mujeres. Esta opresión se expresa desde gestos como el salón, el jardín y la cocina, donde el acceso de la mujer se diseña desde adentro (salir a la sala, a la terraza), y el del marido desde afuera (entrar a la sala, llegar a la terraza). Aunque estos espacios vividos responden a modelos que no se pueden generalizar, la concepción espacial de la arquitectura le da la preferencia a ciertos roles y maneras de habitar el espacio, de acuerdo con lo sexuado de las relaciones intrafamiliares y su proyección en el espacio doméstico. Pero, al igual que el espacio urbano, los modelos de dominación masculina se transfieren al ámbito cotidiano de las viviendas, cuyo potencial de conflicto y de violencia deja al descubierto que "la esfera doméstica es todo menos un espacio apacible y celestial" (Kuhlmann, 2013: 174), y que el espacio interior está lejos de ser un mundo de seguridad, de confort y protección para las mujeres.

En contra de los abordajes binarios de exterior-interior y hombremujer, es necesario repensar la arquitectura, dice Rendell, desde "otros [conceptos] menos binarios y jerárquicos como el margen, el intersticio, lo cotidiano, lo heterotópico y lo abyecto" (2011: 28). Se trata, entre otras cosas, de ir más allá de la asignación de roles de género a la práctica profesional del arquitecto, pero utilizar estas dicotomías como un punto de partida para replantear la arquitectura en su conjunto. Es aquí precisamente donde el análisis de la frontera de género pudo convertirse en una herramienta excepcional para replantear los conceptos y las convenciones ad intra de los sistemas de conocimiento y contestar las articulaciones político sociales basadas en el posicionamiento de las mujeres en las estructuras jerárquicas eminentemente patriarcales.

\section{Genealogía de la arquitectura y los roles de género}

La domesticidad es una construcción del siglo XIX, referida a todo un conjunto de ideas desarrolladas en reacción con la división entre el lugar de trabajo y el hogar (Heynen, 2005: 7). Como se mencionó al respecto de la dicotomía exterior/interior - hombre/mujer, la división genérica del espacio resulta en una esfera pública asociada a lo masculino, y un mundo doméstico asociado a lo femenino.

En esta definición tradicional de dos universos, uno definido por el trabajo remunerado, productivo y masculino; y otro por el trabajo doméstico, reproductivo y femenino, encontramos a las mujeres desempeñando labores de servicio para su familia. Actividades como lavar, cocinar, administrar, transportar, acompañar y cuidar de los niños y las personas mayores son invariablemente atribuidos al espacio doméstico y, por lo tanto, a la mujer: "El trabajo reproductivo hace referencia al trabajo destinado a satisfacer las necesidades de la familia. A pesar de constituir una dimensión necesaria para la reproducción de la sociedad, su desarrollo ha quedado históricamente circunscrito al marco privado, 
primordialmente a la esfera doméstica, razón por la cual también se define como "trabajo doméstico" o familiar" (Larraña, 2004: 32).

Encontramos entonces que, si bien los espacios relacionados con lo doméstico se refieren a lo femenino, una vez dentro del hogar son aquellos relacionados con las actividades de trabajo, servicio y cuidados en los que el rol de la mujer es representado: cocinas, cuartos de lavado y tendido, alacenas, cuartos infantiles, etc. Es precisamente la cocina la mayor referencia que podemos encontrar en cuanto a un espacio histórico de significación femenina que, además, evidencia de manera clara las transformaciones sociales y su manifestación directa en la configuración espacial de lo doméstico: "En conjunto, la organización del espacio y la del género podrían cambiar los significados a lo largo del tiempo, de acuerdo con los cambios de circunstancias y de metáforas, que pueden ser comprendidos únicamente en relación con estas" (Hill, 2003: 5).

Si bien, las cocinas son relacionadas con los ideales de hogar, calor, reunión y familia, también son el espacio del trabajo y del subyugo femenino. Basta un vistazo a la literatura mexicana para notarlo: Tita, protagonista de la novela Como agua para chocolate de Laura Esquivel, es presentada al lector como un personaje vinculado con los actos de cocinar y alimentar desde el momento de su nacimiento, sobre la mesa de la cocina y entre los olores de sopa de fideo y especias: "Este inusitado nacimiento determinó el hecho de que Tita sintiera un inmenso amor por la cocina y que la mayor parte de su vida la pasara en ella, prácticamente desde que nació..." (Esquivel, 2016: 5).

Esquivel presenta, a partir de Tita, el personaje principal, un símbolo de la mujer como sanadora y dadora de alimento. Sin embargo, también es el símbolo de la mujer oprimida por una tradición familiar que le niega cumplir sus aspiraciones, y que designa a la cocina como el escenario de una vida limitada a saciar a los demás: "Tita bajó la cabeza y con la misma fuerza con que sus lágrimas cayeron sobre la mesa, así cayó sobre ella su destino. $\mathrm{Y}$ desde ese momento supieron ella y la mesa que no podían modificar ni tantito la dirección de estas fuerzas desconocidas que las obligaban, a la una, a compartir con Tita su sino, recibiendo sus amargas lágrimas desde el momento en que nació, y a la otra a asumir esta absurda determinación" (Esquivel, 2016: 7).

A pesar de que la vida de la mujer resulta tan íntimamente ligada a la cocina, en la vivienda social contemporánea no es la mujer quien decide la configuración del espacio sino el diseñador arquitectónico, quien opera a partir de concepciones previas asignadas para el rol femenino. De esta manera, desde el proyecto de diseño y el establecimiento de tipologías, se asignan los lugares de la vivienda considerando espacios para lo femenino, desde un discurso patriarcal heredado por la tradición y la costumbre. De esta manera, las condicionantes establecidas por la ideología y los modos de vida se transfieren al espacio doméstico mediante las características arquitectónicas que los determinan.

La propia localización de la cocina en la casa responde a estas concepciones ideológicas que ya no sólo representan un espacio de servicio, sino de exclusión: "Por mucho tiempo el confinamiento de 
las mujeres se entendía por su servilidad en el espacio doméstico y el estrés de la suciedad que había que esconder del público (Kulhmann, 2013: 177). Es cierto que muchos arquitectos diseñan principalmente a partir de sus intereses por responder a las condiciones climáticas, la relación de la vivienda con el entorno, así como la funcionalidad de los espacios. No obstante, es necesario rebasar esta mirada objetiva e instrumental del espacio habitable, para incorporar en los procesos de pensamiento y de materialización de la arquitectura, una reflexión más profunda de los procesos socioculturales que atraviesan no solamente la disposición material de la vivienda en términos geográficos sino las técnicas y mecanismos que permiten habitarla de acuerdo con una época y una sociedad particular.

En un análisis del espacio doméstico, Gutiérrez de Velasco realiza un estudio de las condicionantes espaciales de la cocina mexicana como símbolos de género, a partir de la lectura de la casa de sus abuelos maternos y paternos. Se describe la cocina como un espacio pequeño, sin luz y ubicado hasta el fondo del predio: un lugar que se deseaba esconder por su continuo uso, su falta de limpieza, sus múltiples olores y desechos; y en donde laboraban un séquito de mujeres esclavizadas junto con la abuela. Esta cocina aparece también como un lugar segregado, diseñado para el trabajo intenso y duro de las labores culinarias diarias de preparación y limpieza, en el que más que esconder el espacio arquitectónico en sí mismo, había que esconder la vergüenza y poca pulcritud de las labores realizadas por las mujeres (Gutiérrez de Velasco, 2017: 252).

De igual forma, siguiendo con Gutiérrez de Velasco, en la configuración de la casa de los abuelos paternos, de tipo chalet y rodeada de jardines, también había una cocina en la parte posterior del predio, separada de la casa habitación. A pesar de que esta zonificación resulta poco funcional en cuanto a las condiciones climáticas del sitio, "las fuerzas ideológicas de clase y separación de actividades eran más fuertes que la lógica racional y de función” (2017: 255), procurando, una vez más, esconder las actividades de servicio, es decir, la labor de la mujer.

Así, históricamente el discurso de la arquitectura doméstica anticipa lo femenino como una categoría para la configuración de la vivienda, construyendo espacios que dan por sentada su asignación a un rol específico. Estas formar arquitectónicas se corresponden con el cumplimiento de otras actividades opresoras de las mujeres e igualmente predeterminadas por la tradición, desde la consideración de la estructura socioespacial dominante.

\section{Modelos de construcción de género en arquitectura}

Como hemos revisado en los apartados anteriores, la asignación de los roles de género se encuentra íntimamente ligada a las relaciones y representaciones de éstos en el espacio. En este entendido, la arquitectura contribuye a una asignación determinista en la cual el rol de lo femenino queda oprimido por las consideraciones tradicionales que le estipulan cumplir ciertas actividades, responsabilidades y actitudes. 
"Las instituciones sirven para disciplinar las mentes, los cuerpos y las emociones, de acuerdo con las relaciones jerárquicas que les son propias, tal como la clase social o el estatus de salud y religioso. El poder se ejerce a través de relaciones clasificatorias de los cuerpos" (Hill, 2003: 16). En este caso la relación jerárquica es el rol de mujer, la institución: la arquitectura.

Particularmente en el ambiente doméstico encontramos narrativas y discursos ideológicos que se reflejan de forma directa en la asignación de lo femenino por medio de las configuraciones arquitectónicas: desde la decoración de interiores hasta la localización histórica de un elemento tan significativo como es la cocina. Es en estos espacios donde es notoria una clasificación excluyente en que los espacios femeninos se ven segregados de manera tradicional: "La exclusión de las mujeres empieza en casa. Después de todo, el vínculo espacial con la casa, por lo general unido a la dependencia económica de la pareja o marido, conlleva a una muy reducida influencia social" (Kulhmann, 2013: 175).

Es por esto que resulta de especial interés el análisis de la vivienda contemporánea como una manifestación de un sistema subyacente, un discurso de poder en que la distribución de los espacios no es más que una forma más de estructurar de acuerdo al sistema normativo existente. "Las consecuencias son particularmente profundas porque la arquitectura y el entorno construido son producto de estrategias (conscientes e inconscientes) dirigidas a la satisfacción de intereses materiales y simbólicos y puestas a disposición a una condición social y económica, y política" (Hill, 2003: 6).

Ante la valoración de la vivienda como una declaración espacial de una serie de ideologías sociales y por la inminencia del desarrollo de la vivienda social ante el crecimiento poblacional, se analizarán casos de conjuntos habitacionales en zonas metropolitanas de acuerdo con los criterios del Diagnóstico de Diseño Arquitectónico y Urbano del Cluster de Vivienda de León, Guanajuato: "Los desarrollos habitacionales analizados cuentan con fecha de venta a partir de 2013 y un costo que va de 250 000 hasta 350000 pesos, en proceso de construcción y sin entrega al municipio". (Cluster de Vivienda, 2015). Si bien dicho criterio responde a los objetivos propios del Diagnóstico citado, los casos de estudio resultan efectivos para la presente investigación desde la consideración de la gran franja poblacional que accede a este tipo de viviendas, quedando sujetas a una configuración predeterminada por un sistema de construcción en serie que, a su vez, responde a consideraciones a priori de los usos y modos de vida por parte de los diseñadores y constructores. 


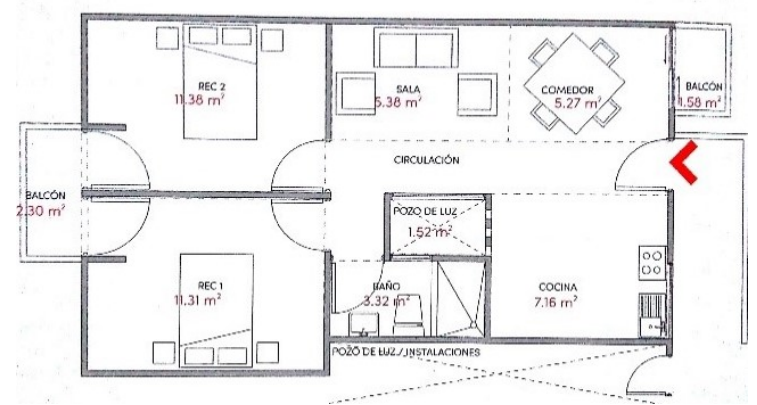

Figura 1. Planta tipo del Conjunto residencial de Cañada del Real en León, Guanajuato. Diagnóstico de diseño arquitectónico y urbano. Fuente: Cluster de Vivienda (2015: 44).

En la planta tipo del departamento de $56 \mathrm{~m}^{2}$, llama la atención en primer lugar la nueva localización de la cocina en la vivienda contemporánea. Si bien, en el recorrido histórico previamente desarrollado las cocinas se encontraban relegadas para ocultar un espacio de "trabajo sucio", en este caso en particular la cocina se ve situada justo junto al acceso principal de la vivienda, sin haber si quiera muros que impidan la visibilidad de la misma (figura 1).

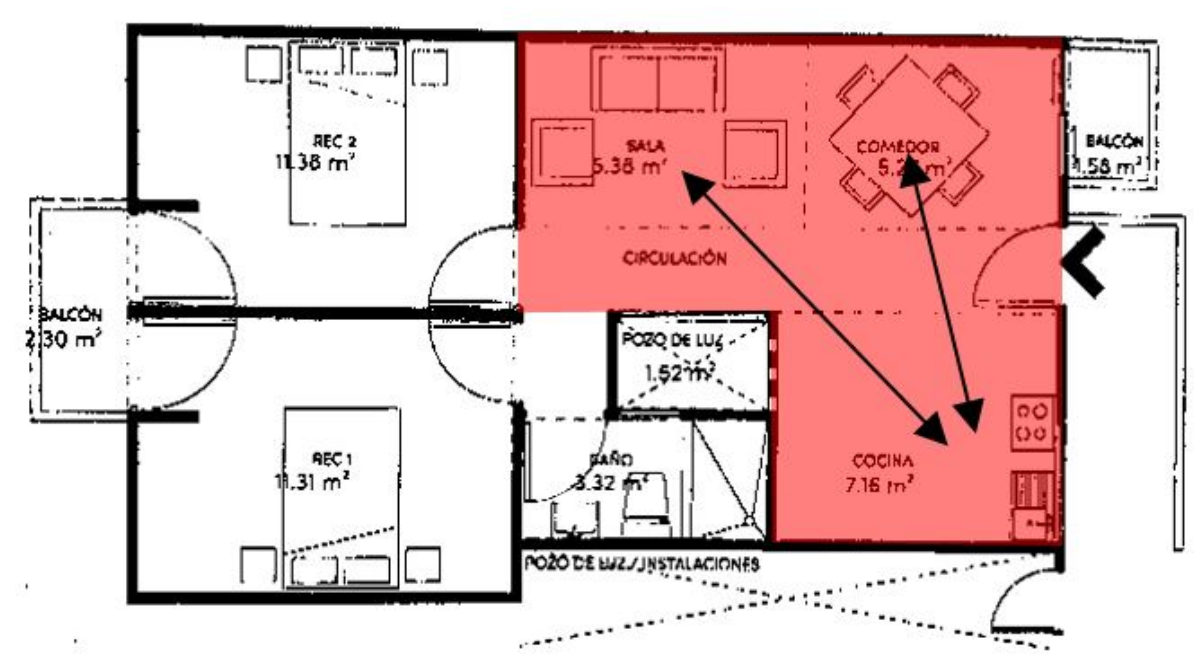

Figura 2. Relación de espacios domésticos.

Fuente: Elaboración propia.

El espacio se ha reformulado ante nuevas premisas: por un lado, el uso de la tecnología. Se presupone, por ejemplo, que una cocina con estufa de gas o eléctrica será menos sucia (menos "ocultable") que un fogón de leña o carbón. Pero, sobre todo, esta nueva disposición espacial nos lleva a pensar a una necesidad de visibilidad desde la cocina, en la que la persona -la mujer- que trabaja en ella pueda tener un control de las otras actividades del resto de los espacios públicos al menos. La mujer ha sido constantemente ligada a la idea y ejecución del multitasking, por lo que le es demandado ya no sólo cumplir con una serie de actividades domésticas, sino el cumplirlas todas al mismo tiempo (figura 2). 
Segundo caso: Conjunto habitacional Las Anacuas en Monterrey, Nuevo León

En este ejemplo de vivienda, de $40 \mathrm{~m}^{2}$ construidos en el momento de entrega, la cocina es nuevamente colocada al fondo de la vivienda, pero no en un sentido de segregación sino por el contrario, como el principal remate visual al acceder a la casa. Una vez más, la funcionalidad del espacio y la fe en la pulcritud a la que los aparatos domésticos nos permiten aspirar (ambas características heredadas de la modernidad) determinan una cocina abierta al resto de la zona pública (figura 3).

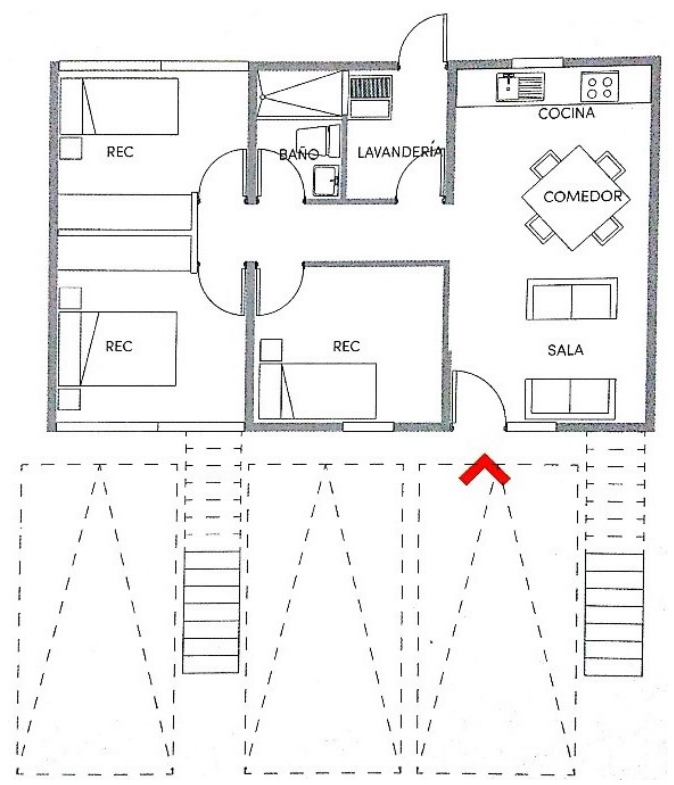

Figura 3. Planta tipo del Conjunto habitacional Las Anacuas en Monterrey,

Nuevo León. En Diagnóstico de diseño arquitectónico y urbano.

Fuente: Cluster de Vivienda (2015: 183).

Más evidente aún resulta, continuando con el tema de la visibilidad del espacio doméstico, la capacidad de vigilancia ejercida desde el interior femenino del mismo, la propia cocina. A esto se le añade la presencia del espacio de lavandería, estratégicamente acoplado como un área de servicio/trabajo más para estar pendiente y que, junto con el área de baño, separan del área privada/descanso del ajetreo de la labor doméstica (figura 4). 


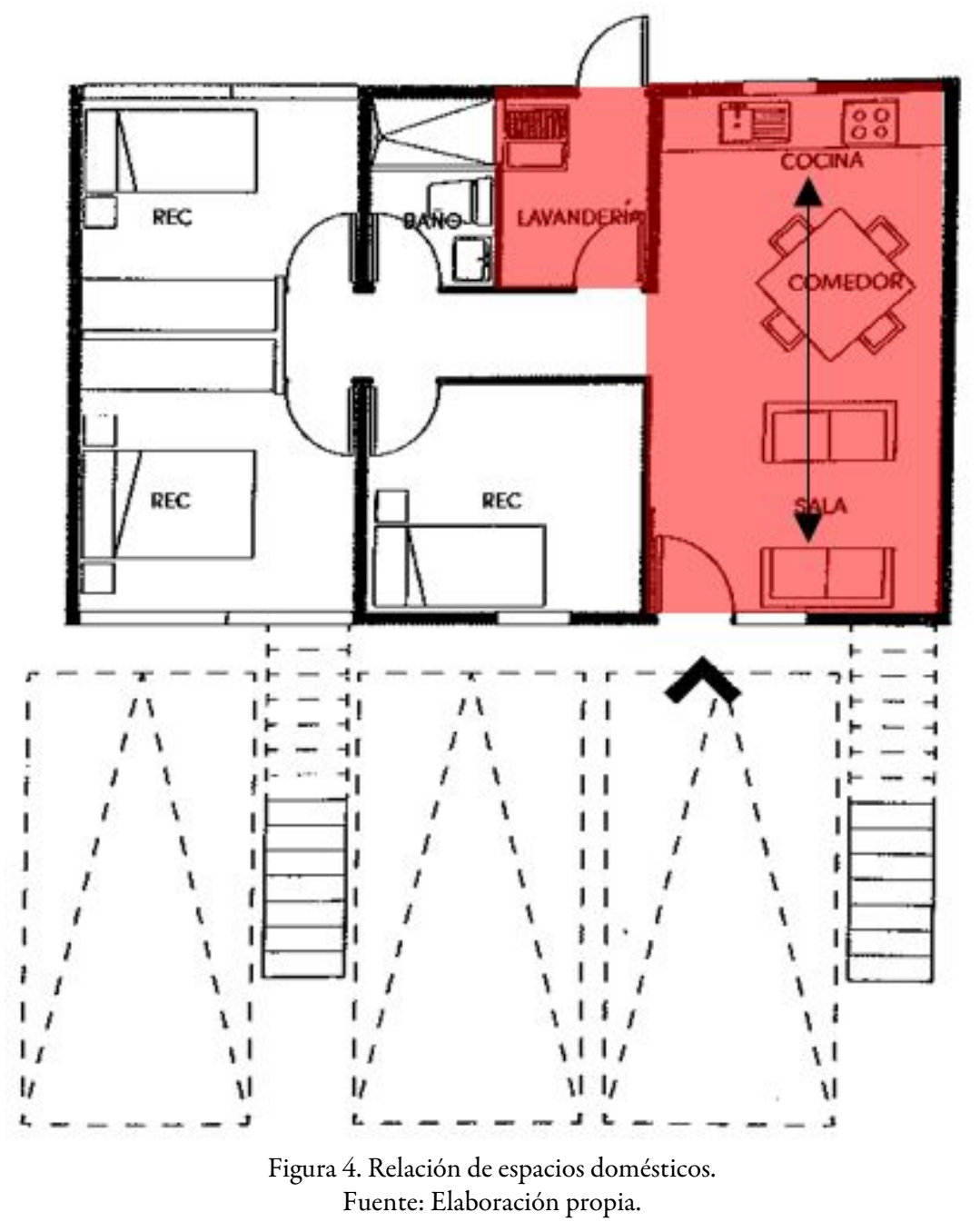


Tercer caso: Desarrollo habitacional Real Solare en El Marqués, Querétaro

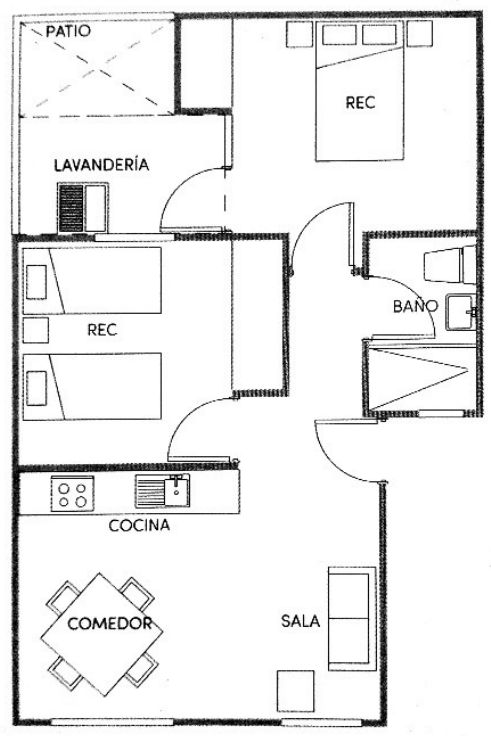

Figura 5. Planta tipo del Desarrollo habitacional Real Solare en El Marqués, Querétaro. En Diagnóstico de diseño arquitectónico y urbano. Fuente: Cluster de Vivienda (2015: 190).

La vivienda de este ejemplo cuenta con $47 \mathrm{~m}^{2}$, desarrollados desde una zonificación en que, si bien la cocina no aparece directamente junto al acceso o como remate visual desde el mismo, sí resulta visible y sí mantiene vigilancia-servicio simultáneamente sobre las áreas de comedor y sala (figura 5). 


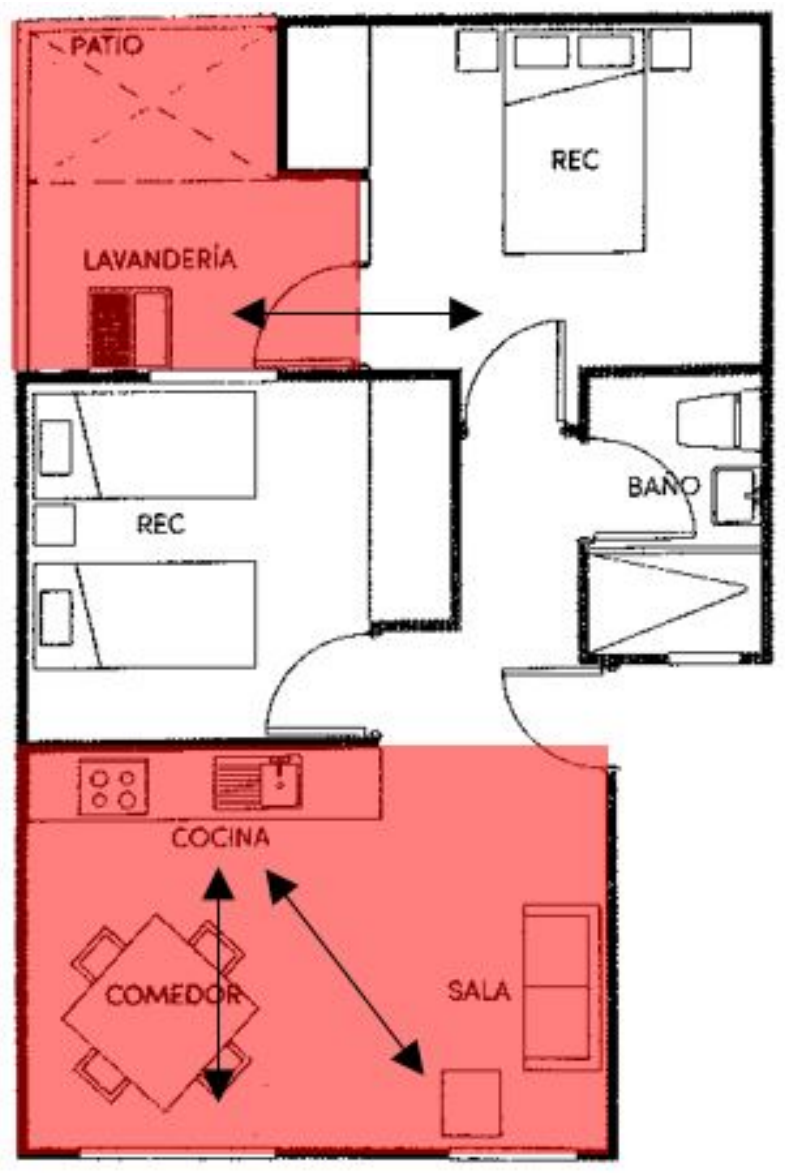

Figura 6. Relación de espacios domésticos. Fuente: Elaboración propia.

La consideración que podría resultar extraña o incluso verse como una disposición incómoda es la colocación del área de lavado y tendido directamente conectada con una de las recámaras (figura 6). Esta situación podría parecernos una invasión a la privacidad por parte de la actividad doméstica y un inconveniente para los habitantes, siempre en el entendido de que una parte de ellos se encuentra en tranquilo reposo en sus habitaciones, mientras las actividades domésticas, femeninas, se deben excluir para realizarse lejos de esa esfera. Sin embargo, al analizar este caso en particular se abre la posibilidad a un entendimiento diferente de la actividad doméstica, en la que todos los espacios y habitantes están involucrados.

Si bien no es la intención defender o criticar los programas arquitectónicos y zonificaciones particulares de los casos estudiados, es útil como ejercicio de observación acerca de las decisiones de diseño arquitectónico que son tomadas simplemente porque deben ser así. Esto nos remite una vez más al pensamiento de Judith Butler, "lo que tomamos como un rasgo interno es en realidad algo que anticipamos y producimos" (Butler, 1990: 17). ¿No es esto lo que sucede con la arquitectura al construir espacios para que funcionen de la forma en que damos por hecho que deberían funcionar? Dotar de roles de género el espacio es un ejercicio inconsciente ante el que conviene poner atención para no ser partícipes de un sistema de dominación que, si bien ha 
evolucionado con los nuevos modos de vida, sigue haciéndose presente en el proyecto arquitectónico.

\section{CONCLUSIONES}

La relación entre la arquitectura y la frontera trazada entre hombres y mujeres es uno de tantos mecanismos societales para la ratificación de la dominación masculina por medio de la asignación de roles y el mantenimiento de las diferencias entre sexos. El papel de la arquitectura es fundamental para la construcción de los roles de género y la externalización de los discursos que organizan el espacio a partir de las diferencias entre hombres y mujeres. Más allá del análisis de los sistemas de dominación en nuevos territorios como el espacio doméstico, es importante que, a semejanza de las nuevas configuraciones de las familias contemporáneas (monoparental, sin hijos, padres separados, compuesta, extensa), se propongan también nuevos planteamientos de los programas arquitectónicos convencionales, con una reinterpretación del espacio como una alternativa a los mecanismos de opresión y violencia determinados por las prácticas tradicionales del diseño arquitectónico. Este nuevo enfoque abre la posibilidad de presentar a una arquitectura con un nuevo papel de experimentación y apertura, versus una arquitectura entendida como práctica reglamentadora, que inscribe las diferencias de género para ratificar el orden político y social.

En tanto que la casa mexicana contemporánea se siga pensando, diseñando y materializando como un espacio femenino, también el control y la opresión de las mujeres seguirán ratificándose en la arquitectura. Los sistemas de dominación materializados en el espacio arquitectónico no solamente reproducen la diferencia de roles, sino que abonan para que los roles asignados en la habitabilidad cotidiana del espacio doméstico se profundicen y se mantengan. De aquí que el espacio edificado tenga un mayor alcance en la reproducción de las desiguales entre hombres y mujeres, que las constantes diferencias buscadas en la representación de los cuerpos en el arte, o las críticas de la menor presencia de mujeres en la farándula de la arquitectura contemporánea.

\section{FUENTES DE CONSULTA}

Butler, J. (1990), El género en disputa, Paidós, Barcelona.

Cluster de Vivienda (2015), Diagnóstico de Diseño Arquitectónico y Urbano, Cluster de Vivienda, León.

Esquivel, L. (2016), Como agua para chocolate, Debolsillo, México.

Gutiérrez de Velasco, E. (2017), Las cocinas mexicanas a través de la historia, su localización, atributos y su relación con la ciudad: símbolos de género y vida cotidiana. Memorias del Segundo Congreso Internacional sobre Género y Espacio y Tercer Semanario Latino-Americano de Geografía, Género e Sexualidades, Universidad Autónoma Metropolitana, México.

Hills, H. (2003), "Theorizing the relationships between architecture and gender in Early Modern Europe". En Hills, Helen (ed.), Architecture and the 
politics of gender in Early Modern Europe, ASHGATE, Inglaterra, pp. 3-22.

Heynen, H. (2005), “Modernity and domesticity: tensions and contradictions". In Heynen, Hilde y Gülsüm Baydar (eds.), Negociating domesticity: Spatial productions of gender in modern architecture, Routledge, Nueva York, pp. 1-29.

Kuhlmann, D. (2013), Gender studies in architecture: space, power and differenc, Routledge, Nueva York.

Larrañaga, I., Arregi, B. \& Arpal, J. (2004), "El trabajo reproductivo o doméstico", Gaceta Sanitaria, 18(1), pp. 31-37.

Lawrence, R. (1982), "L'espace domestique: typologie et vécu”, Cahiers Internationaux de Sociologie, no. 72, pp. 55-75.

Martínez-López, C. (2016), "Matronazgo, arquitectura y redes de poder". En Martínez-López, Cándida y Felipe Serrano Estrella (eds.), Matronazgo y arquitectura: de la antigüedad a la Edad Moderna, Granada, EUG, pp. 11-26.

Novas, M. (2014), Arquitectura y género, una reflexión teórica, Estudio Xarda, Bueu.

Rendell, J. (2011), "Critical spatial practices: Setting out a feminist approach to some modes and what matters in architecture". En Brown, Lori A. (ed.), Feminist practices: interdisciplinary approaches to women in architecture, ASHGATE, Inglaterra.

Ríos-Llamas, C. (2018a), “Agorafobia en México y Francia: de miedos urbanos y vidas privatizadas", Espacialidades, revista de temas contemporáneos sobre lugares, política y cultura, 8(1), pp. 39-51.

Ríos-Llamas, C. (2018b), Ciudades obesogénicas y mujeres vulnerables, ITESO, México.

Vigarello, G. (2005a), Corregir el cuerpo: historia de un poder pedagógico, Nueva visión, Buenos Aires.

Vigarello, G. (2005b), Historia de la belleza, Nueva visión, Buenos Aires.

Vigarello, G. (1999), Lo sano y lo malsano: historia de las prácticas de la salud desde la Edad Media hasta nuestros días, Abada, Madrid.

Vigarello, G. (1998), Historia de la violación, siglos XVI-XX, Cátedra, Madrid.

Vigarello, G. (1991), Lo limpio y lo sucio. La higiene del cuerpo desde la Edad Media, Alianza, Madrid.

\section{Notas}

[1] Conviene mencionar la gran influencia de los trabajos de Georges Vigarello sobre la representación del cuerpo en las diferentes épocas y las distinciones entre la feminidad y la masculinidad de las corporalidades. Esto se puede constatar en sus obras como Corregir el cuerpo: historia de un poder pedagógico (2005a [1978]), Lo limpio y lo sucio. La higiene del cuerpo desde la Edad Media (1991[1985]), Lo sano y lo malsano: historia de las prácticas de la salud desde la Edad Media hasta nuestros dias (1999[1993]), Historia de la violación, siglos XVI-XX (1998), e Historia de la belleza (2005b [2004]). 